\title{
Le monde en révolutions ou le parcours désorientant de M. N. Roy
}

Nicola Pozza

\section{(2) OpenEdition}

1 Journals

Édition électronique

URL : http://journals.openedition.org/edl/785

DOI : $10.4000 /$ edl. 785

ISSN : 2296-5084

Éditeur

Université de Lausanne

\section{Édition imprimée}

Date de publication : 15 septembre 2014

Pagination : 343-366

ISBN : 978-2-940331-35-2

ISSN : 0014-2026

\section{Référence électronique}

Nicola Pozza, "Le monde en révolutions ou le parcours désorientant de M. N. Roy », Études de lettres [En ligne], 2-3 | 2014, mis en ligne le 15 septembre 2017, consulté le 20 décembre 2020. URL : http:// journals.openedition.org/edl/785; DOI : https://doi.org/10.4000/edl.785 


\section{LE MONDE EN RÉVOLUTIONS OU LE PARCOURS DÉSORIENTANT DE M. N. ROY}

Le parcours de Manabendra Nath Roy (1887-1954) est déroutant à plus d'un titre, car l'auteur bengali s'est constamment joué des frontières établies, tant nationales et géographiques qu'idéologiques et partisanes. Acteur central de la naissance du Parti communiste du Mexique (1919) puis fondateur du Parti communiste indien (1920), proche de Lénine et membre influent de l'Internationale communiste, Roy s'est par la suite complètement distancié de la doctrine communiste puis de la politique du Congress pour fonder en 1940 un mouvement dissident, le Radical Democratic Party, avant de remplacer ce dernier par le Radical Humanist Movement (en 1948). Sans cesse en déplacement, toujours distant vis-à-vis des avis dominants, il s'est particulièrement opposé au leitmotiv orientaliste de l'époque qui cherchait à glorifier «l'héritage spirituel de l'Inde", en prônant au contraire une philosophie matérialiste puis humaniste. Nous analyserons dans cet article la position de M. N. Roy par rapport aux discours orientalistes et panasiatistes de son époque et examinerons notamment sa position très critique à l'égard de l'Inde conçue comme antithèse spirituelle à l'Europe. Ceci nous permettra d'interroger la paire antagonique formée par les notions de «centre» et de «marges» (ou "périphérie»), en utilisant le double parcours biographique et idéologique de l'auteur comme cas d'étude.

\section{Introduction}

Le parcours de Manabendra Nath Roy (1887-1954) est déroutant à plus d'un titre, car l'auteur bengali s'est constamment joué des frontières établies, tant nationales et géographiques qu'idéologiques et partisanes. Acteur central de la naissance du Parti communiste du Mexique (1919) puis fondateur du Parti communiste indien (1920), proche de Lénine et membre influent de l'Internationale communiste, Roy s'est par la suite complètement distancié de la doctrine communiste puis de la politique 
du Congress pour fonder en 1940 un mouvement dissident, le Radical Democratic Party, avant de remplacer ce dernier par le Radical Humanist Movement (en 1948). Sans cesse en déplacement, toujours distant visà-vis des avis dominants, il s'est particulièrement opposé au leitmotiv orientaliste de l'époque qui cherchait à glorifier «l'héritage spirituel de l'Inde", en prônant au contraire une philosophie matérialiste puis humaniste. Contrairement à la majorité de ses compatriotes, Roy ne basa ainsi pas sa lutte contre l'impérialisme colonial sur le modèle du nationalisme, mais l'inscrivit au contraire dans la perspective d'une solidarité internationale, qu'il voulait concomitante à tous les mouvements révolutionnaires. Le mouvement de libération de l'Inde devait participer selon lui d'un mouvement universel, puisque la question de la libération était à ses yeux avant tout une question de lutte des classes, dont la pertinence sociale et historique se devait d'être globale, débordant le cadre limité des frontières nationales.

Dans ces circonstances, examiner le parcours de Roy selon la polarité "centre vs périphérie" parait, de prime abord en tout cas, peu pertinent. Face à son parcours sinueux, autant d'un point de vue intellectuel que géographique, la question se pose dès lors de savoir où et comment situer le "local", où placer les "marges", et par rapport à quel centre (pour reprendre les termes de l'argumentaire de cette publication)? Comment peut-on situer Roy dans cet espace et par rapport à cette polarité? Réciproquement, son parcours mouvant, "désorientant», permet-il de questionner et de repenser cette polarité? C'est ce que cette contribution se propose d'examiner en retraçant, dans un premier temps, les grandes étapes du parcours géographique et politique de Roy. Il ne sera pas fait mention de tous ses faits et gestes ${ }^{1}$, mais nous insisterons sur certaines étapes et certains aspects capitaux de son parcours, en vue d'apporter un premier élément de réponse aux questions soulevées ci-dessus et afin de souligner l'extrême cosmopolitanisme du personnage, loin de l'unique relation binaire Inde-Europe. Dans un deuxième temps, l'analyse portera sur quelques textes qui permettront de mettre en perspective et de clarifier le parcours et les prises de position idéologiques de l'auteur.

I. Pour de plus amples informations, voir M. N. Roy, Selected Works of M. N. Roy, vol. 1, p. 9-56; J. P. Haithcox, Communism and Nationalism in India; V. B. Karnik, M. N. Roy: Political Biography. 
Cette contribution se limitera à la première partie des œuvres de Roy, à savoir à sa période «marxiste».

\section{Biographie: un parcours sinueux}

Né Narendra Nath Bhattacharya au sein d'une famille de brahmanes du Bengale, Roy s'est dès son plus jeune âge profilé comme un révolutionnaire libre-penseur. Provenant d'un milieu éduqué - son père était enseignant de sanskrit -, il fut également influencé par l'intense atmosphère intellectuelle et réformatrice du Bengale de cette fin de XIX ${ }^{\mathrm{e}}$ siècle. Roy abandonna rapidement l'école pour se consacrer pleinement au mouvement de résistance bengali, le mouvement Swadeshi, durant les années 1906 à 1914.

C'est dans ce contexte révolutionnaire et suite à ces années de formation que Roy réalise en 1915 plusieurs aller-retour entre Jakarta et Kolkata (Calcutta) afin de livrer des armes au mouvement Swadeshi, grâce à l'aide des autorités allemandes basées dans le Sud-Est asiatique ${ }^{2}$. La même année, il décide de se rendre à Berlin, en utilisant un passeport franco-indien fourni par l'ambassade d'Allemagne à Pékin et en voyageant sous le nom de "Père Martin". Il se dirige tout d'abord vers Shanghai ${ }^{3}$, puis embarque sur un bateau de croisière japonais à destination de San Francisco, qu'il atteint en juin 1916. Fuyant la police britannique, il se dirige immédiatement à Palo Alto $(\mathrm{Ca})$, où il adopte son nouveau nom de Manabendra Nath Roy ${ }^{4}$ et où il fait la connaissance d'une étudiante de la Stanford University, Evelyn Trent (18921970, connue par la suite sous le nom de Shanti Devi), qui deviendra

2. Cf. S. Bhattacharya, «Bengal and Germany», p. 6: «It may be mentioned here that around the same time (1915-1916) Germany's involvement with the Indian nationalist movement reached it's [sic] climax. Under the leadership of Baron Openheimer, the German Foreign office opened a special committee for the Orient (Turkey) and India.»

3. En passant par la Malaisie, l'Indonésie, l'Indochine, les Philippines, le Japon, la Corée et la Chine.

4. Il opère ce changement de nom sur le conseil de Dhana Gopal Mukherjee, l'ami chez qui il loge, qui lui recommande de faire ainsi table rase de "son passé de militant nationaliste pour se consacrer à la révolution sociale» (V. B. Karnik, M. N. Roy: Political Biography, p. 41). 
sa première épouse et son mentor en matière d'éducation politique. En octobre, il part pour New York où il sympathise avec la diaspora indienne et le mouvement anticolonial et où il rencontre le célèbre leader nationaliste Lala Lajpat Rai (1865-1928). C’est là qu'il se met à étudier sérieusement le marxisme dans l'idée première de le combattre; il finira au contraire par l'adopter et par en faire l'idéologie guidant tous ses écrits pour les années à venir. En juin 1917, il fuit les Etats-Unis pour Mexico City, où il apprend très vite l'espagnol (au point d'écrire et de publier ses propres textes en espagnol l'année suivante déjà) - il s'initie également à cette occasion à l'allemand et au français ${ }^{5}$. Il sympathise avec le président mexicain Venustiano Carranza et la cause socialiste. En décembre de l'année suivante, il est nommé secrétaire général du Parti socialiste du Mexique, qu'il transforme le 28 novembre 1919 en Parti communiste du Mexique ${ }^{6}$, suite à sa rencontre avec Mikhaïl Borodine (1884-1951), un émissaire du Komintern, et à leur amitié naissante. Ce dernier l'invite alors à participer au deuxième Congrès de l'Internationale communiste qui se tiendra à Moscou du 19 juillet au 7 août 1920 .

Roy part pour l'Europe, débarque en Espagne puis s'arrête en chemin à Zurich et à Berlin avant de se rendre à Moscou pour le Congrès. A l'invitation de Lénine (1870-1924) et en réponse aux «Thèses sur les questions nationale et coloniale» de ce dernier, il y présente ses "Thèses complémentaires" - thèses qui seront adoptées, moyennant quelques modifications répondant aux points de divergences existant entre sa position et celle du révolutionnaire russe ${ }^{7}$. Roy estime ainsi, contrairement à Lénine, que la bourgeoisie indienne, de nature réactionnaire, ne peut en aucun cas être une alliée du mouvement révolutionnaire, car elle ne viserait au final qu'à se substituer aux "exploiteurs étrangers ».

5. Cf. J. Vigreux, «Manabendra Nath Roy (1887-1954)», version électronique $\$ 10$ : «Roy met à profit ce séjour pour parfaire sa maîtrise des langues; polyglotte, il parle et écrit en bengali, en anglais, en espagnol, en allemand, en français et en russe. C'est au Mexique, qu'il publie son premier livre en espagnol, La India, Su Pasado, Su Presente $Y$ Su Porvenir (L'Inde, son passé, son présent et son avenir) dans lequel il développe ses thèses anticolonialistes." Sur cet épisode mexicain, voir M. Goebel, «Una biografía entre espacios".

6. Sur cet épisode, voir B. Carr, «Marxism and Anarchism in the Formation of the Mexican Communist Party, 1910-19».

7. Dans sa version française, le texte final sera publié sous le titre de "Thèses et additions sur les questions nationale et coloniale». 
En bref, pour reprendre le résumé de Sibnarayan Ray, éditeur et auteur de l'Introduction au premier volume des Selected Works of M. N. Roy, le révolutionnaire indien estimait que:

(a) a revolution in Europe depended on the course of the revolution in Asia bringing about the break-up of the colonial empires; $(b)$ in the colonies, especially India, there were two movements, one bourgeois democratic, limited to the middle classes, which sought compromise with the existing order, the other a mass movement which represented the exploited majority for whom political independence to be achieved and made meaningful required at the same time a social and economic revolution; and $(c)$ the Comintern should support revolutionary mass movements and not the colonial middle class movements and leaderships ${ }^{8}$.

Immédiatement après ce Congrès intervient une étape centrale, autant d'un point de vue symbolique et géographique par rapport au thème de cet ouvrage que d'un point de vue historique par rapport au développement du communisme en Inde. Roy part avec sa femme pour Tachkent - alors capitale de la République Socialiste Soviétique Autonome du Turkestan -, afin de monter une armée de libération formée de musulmans venus d'Inde dans le cadre du mouvement du Califat ${ }^{9}$. Sur place, il fonde le 17 octobre 1920, avec sa femme et cinq autres membres fondateurs, le "Parti communiste de l'Inde», qui adopte les principes de l'Internationale communiste $^{10}$. Il retourne à Moscou en mai 1921, avant

8. M. N. Roy, Selected Works of M. N. Roy, vol. 1, p. 20.

9. Le mouvement du Califat (ou Khilafat en Inde) fut un mouvement qui prit place en Inde en 1919 pour soutenir le sultan (et calife) de l'Empire ottoman et protester contre la politique britannique au Moyen-Orient. Suite au traité de Sèvres, qui signait le démembrement de l'Empire ottoman, le Central Khilafat Committee vit le jour à Allahabad en juin 1920 et inspira la première campagne de non-coopération (19201922), pour laquelle l'engagement de Gandhi allait s'avérer crucial. Sur le mouvement du Califat, voir G. Minault, The Khilafat Movement; M. N. Qureshi, Pan-Islam in British Indian Politics. Sur le rôle de Roy à Tachkent, voir G. Boquerat, «Du bond en avant au retour en arrière, évolution de la perception indienne de l'Asie centrale au cours du XX $\mathrm{X}^{\mathrm{e}}$ siècle», p. 286; J. P. Haithcox, Communism and Nationalism in India, p. 20-23.

IO. Le procès-verbal de la rencontre est reproduit dans M. N. Roy, Selected Works of M. N. Roy, vol. 1, p. 179. Les communistes indiens actuels sont divisés sur la question de l'origine du parti en Inde. Les membres du Parti communiste de l'Inde (marxiste) soutiennent qu'il a justement été fondé à Tachkent en 1920, alors que ceux du Parti 
d'aller s'établir en avril 1922 à Berlin où il devient un des proches de August Thalheimer (1884-1948) et de Heinrich Brandler (1881-1967), leaders de la Ligue spartakiste (Spartakus Bund). Cette année-là permet d'illustrer l'activité incessante et l'énergie inépuisable de Roy: il arrive en avril à Berlin, sort le 15 mai le premier numéro de son nouveau journal, The Vanguard of Indian Independence, et publie avant la fin de l'année trois livres: India in Transition (son premier livre majeur, qui va être utilisé ci-après comme principale source d'analyse), India's Problem and its Solution, et What Do We Want? ${ }^{11}$.

Roy devient alors le principal exégète du marxisme sur la question coloniale. D'ici à fin 1926, il fait partie de tous les organes centraux de l'Internationale communiste: le Présidium, le Secrétariat politique, le Comité exécutif et le Congrès Mondial. A ce titre, mais aussi parce qu'il cherche à fuir les autorités britanniques, il voyage constamment à travers l'Europe, tout en étant essentiellement basé à Berlin ${ }^{12}$. Mais en raison de sa position critique vis-à-vis de la centralisation moscovite et de la «bolchevisation" en cours, ainsi que de son soutien au Parti communiste allemand de l'opposition (KPD-O), Roy se fait officiellement exclure de l'Internationale communiste en décembre $1929^{13}$. Il est alors traité de «renégat», de "traître», de "droitier»-critiques qui lui seront aussi adressées plus tard par les membres du Parti communiste indien.

communiste de l'Inde (CPI) font remonter sa naissance au 26 décembre 1925 à l'occasion de la première conférence communiste panindienne à Kanpur.

II. J. P. Haithcox, Communism and Nationalism in India, p. 24.

I2. J. Vigreux, «Manabendra Nath Roy (1887-1954)», version électronique $\$ 20$.

13. Sur les raisons de cette expulsion, voir J. P. Haithcox, Communism and Nationalism in India, p. 130-139. V. B. Karnik, un de ses fidèles alliés du Radical Humanist Movement, résume ainsi les critiques que Roy formulait alors contre le communisme tel qu'il le percevait: "Communism, which he had hoped would after its victorious fight against fascism assume the leadership of world forces of progress, had degenerated into nationalism and equated itself with the nationalist interests of Russia.» (M. N. Roy: Political Biography, p. 543). Avant cela toutefois, en février 1927, Roy aura été envoyé à Guangzhou (Canton) comme représentant du Komintern pour soutenir le mouvement révolutionnaire chinois (et le soulèvement agraire contre le nationalisme du Guomindang de Tchang Kaï-chek). Après l'échec du mouvement agraire, Roy quitte la Chine en juillet et se tourne vers une autre approche de la solidarité sociale, estimant que la paysannerie seule ne peut mener la révolution et se libérer: il propose de former le Workers and Peasants Parties (WPP) en Inde, qui inclut cette fois la petite bourgeoisie. 
En décembre 1930, il retourne en Inde, à Mumbai (Bombay), alors centre de la contestation ouvrière indienne. Il est emprisonné peu après, suite au procès de Kanpur qui l'avait condamné six ans plus tôt pour conspiration contre l'Empire britannique. Il met à profit ses années de détention (du 21 juillet 1931 au 20 novembre 1936) pour rédiger plus de 3000 pages de manuscrits (appelés "The Prison Manuscripts»), contenant ses réflexions sur le matérialisme historique et le rationalisme, sur la situation en Inde, mais aussi sur les développements de la physique ${ }^{14}$.

A sa sortie de prison, il rejoint l'Indian National Congress. Ellen Gottschalk (1904-1961), qu'il a connue à Berlin et qui deviendra sa seconde épouse, le rejoint quelques mois plus tard. Ils s'installent à Dehra Dun, au pied de l'Himalaya, où ils organisent tous les deux ans des camps d'études, de 1940 à 1950. En 1939, suite à la non-élection de Subhas Chandra Bose (1897-1945) au poste de président du Congress, Roy rompt avec ce parti et fonde un groupe dissident, la League of Radical Congressmen. L'année suivante, à l'occasion du premier "anniversaire» du début de la Seconde Guerre mondiale, cette même League organise une manifestation antifasciste. Dès le début de la guerre, Roy estimait en effet que le véritable danger pour la démocratie indienne était dorénavant le fascisme et non plus l'impérialisme. Il s'aliène les communistes et les congressistes et se fait expulser du Congress qui refusait tout soutien aux Britanniques contre l'Allemagne nazie. Roy décide dans ces conditions de fonder un nouveau parti politique indépendant, le Radical Democratic Party ${ }^{15}$.

En 1946, il prépare pour la réunion de son parti à Mumbai une série de principes de base, qui seront connus et publiés en 1947 sous le nom de New Humanism - A Manifesto. En décembre 1948, suite à l'échec du

I4. Cf. R. Nath, «Manbendra Nath Roy (1887-1954)»: «Roy tried to reformulate materialism in the light of latest developments in the physical and biological sciences. He was convinced that without the growth and development of a materialist and rationalist outlook in India, neither a renaissance nor a democratic revolution would be possible.»

I5. Par antipathie à l'égard des institutions et par rejet de ses pairs, Roy s'aliène de plus en plus d'anciens «amis» communistes ou congressistes. Contrairement à eux, il est alors en faveur d'une «mediated autonomy, in which freedom would arrive within the framework of a South Asian federative union of member nations, and within a transnational federation of democratic governments worldwide" (K. Manjapra, M. N. Roy, p. XVII). 
parti aux élections pour l'Assemblée constituante, il dissout le Radical Democratic Party, estimant par la même occasion que l'existence d'un parti n'est plus compatible avec sa vision de la démocratie. Il fonde le Radical Humanist Movement, dont les valeurs clés se veulent être la liberté, l'humanisme, la raison, la paix et le «matérialisme moniste». C'est à ce moment-là qu'il commence la rédaction de son magnum opus: Reason, Romanticism and Revolution (1952).

L'année suivante, il rebaptise son ancien journal Independent India en The Radical Humanist et change le Marxian Way Quaterly en Humanist Way, soulignant ainsi l'emphase accordée à la notion d'humanisme et son éloignement de la doctrine marxiste. Il établit encore l'Indian Renaissance Institute à Dehra Dun, avant de décéder le 25 janvier 1954 dans cette même ville des suites d'une série d'infarctus.

De ce parcours complexe et sinueux, les points suivants doivent retenir notre attention dans le cadre de cet article. Tout d'abord, le couple notionnel "centre-marge" est difficilement opérant dans le cas de Roy. Bien que le pouvoir britannique - qui peut être perçu pour l'époque coloniale comme le centre par excellence - reste présent en arrière-plan de la lutte idéologique de Roy, il ne constitue pas pour autant chez lui le principal pôle antagonique à l'Inde, ce pôle d'attraction ou de répulsion inspirant la grande majorité de ses contemporains. Chez Roy, un autre pôle intervient dans le tableau, représenté par (au moins) deux "lieux». Un premier, géographique: l'Europe communiste, avec Berlin comme principal centre d'action pour Roy, en décalage avec Moscou, centre dominant du communisme. Un second, idéologique: le marxisme, qui permet à Roy de se positionner en dehors de l'opposition impérialisme vs nationalisme.

Ensuite, le parcours de Roy permet de rappeler que les centres d'action ne se limitaient pas à Londres et Kolkata (puis Delhi pour l'Inde), mais que des régions et des villes apparemment "en marges», comme Tachkent, constituaient elles aussi des centres importants de l'époque.

\section{India in Transition (1922)}

Cette partie traitera essentiellement de son premier essai sur la situation politique et socio-économique de l'Inde contemporaine: India in Transition. Suite à l'adoption en 1920 par l'Internationale communiste 
de ses "Thèses complémentaires", Roy avait eu le sentiment qu'il lui faudrait les développer sous la forme d'une monographie, notamment pour contrebalancer la direction prise par l'Internationale, à son goût trop eurocentrique. Cet essai en est le produit, terminé en octobre 1921 et publié une première fois début 1922 en russe, à Moscou. Après son déménagement à Berlin, Roy publie en avril la version anglaise sous le nom d'un éditeur fictif basé à Genève: Edition de la Librairie J. B. Target. En septembre de cette même année sort la version allemande, publiée à Hambourg, alors qu'au même moment l'édition anglaise est interdite en Inde - les premières copies sont interceptées en août déjà. Rappelons que Roy écrit cet essai pendant le mouvement du Califat et le premier mouvement de non-coopération lancé par Gandhi à la fin de 1920.

Dans ce contexte, les enjeux politiques de la lutte pour l'indépendance de l'Inde sont selon Roy intrinsèquement dépendants des enjeux socio-économiques. Façonnant son discours selon les termes de la rhétorique marxiste ${ }^{16}$, il estime qu'un changement d'ordre politique est inconcevable sans une révolution économique du "prolétariat indien". Idéologiquement, il fait de l'élite économique indienne - la «bourgeoisie nationaliste» ou «réformiste» comme il l'appelle - la cible première de son combat - et non l'impérialisme britannique, cible privilégiée par les nationalistes indiens ${ }^{17}$. Gandhi est doublement au cour de ses critiques,

I6. Voici, à titre d'exemple, le paragraphe final de la version préparatoire de l'Indian Communist Manifesto que Roy rédigea avec sa première femme et Abani Mukherji (1891-1937) à Berlin, peu avant de se rendre au deuxième Congrès de l'Internationale communiste à Moscou: "Cease to fall victims to the imperialist cry that the masses of the East are backward races and must go through the hell fires of capitalistic exploitation from which you are struggling to escape. We appeal to you to recognise the Indian revolutionary movement as a vital part of the world proletarian struggle against capitalism. Help us to raise the banner of the social revolution in India and to free ourselves from capitalistic imperialism that we may help you in the final struggle for the realisation of the universal communist state." (M. N. Roy, Selected Works of M. N. Roy, vol. 1, p. 164). Cette application fidèle du modèle marxiste à la situation indienne, plutôt que son adaptation, a d'ailleurs été régulièrement sujette à critiques; voir par exemple S. Kaviraj, "The Heteronomous Radicalism of M. N. Roy», p. 234, ou A. P. S. Chouhan, D. K. Singh, «M. N. Roy and Marxism», p. 641.

I7. Il devra d'ailleurs clarifier sa critique à l'égard des nationalistes en soulignant la distinction qu'il opère entre «le nationalisme bourgeois conçu pour servir les intérêts des classes supérieures (et notamment les capitalistes) et le patriotisme révolutionnaire 
non seulement comme représentant de cette "bourgeoisie», mais aussi en tant que figure de ce que Roy appelle le "patriotisme spirituel» ${ }^{18}$.

Roy présente dans son introduction à India in Transition les trois points de vue existant alors, selon lui, sur l'Inde et la question coloniale. Il y a tout d'abord, dit-il, celui des «impérialistes britanniques", qui voient l'Inde nouvelle ("the Young India») comme un nouveau-né dont la classe dirigeante britannique serait en quelque sorte le parrain et qui aurait pour "sage-femme» le «libéralisme impérial» ${ }^{19}$. Intervient ensuite le point de vue des "patriotes constitutionnalistes». Ceux-ci correspondent chez Roy aux classes dirigeantes indiennes, qui acceptent et suivent le modèle précédent. Enfin sont définis les «nationalistes orthodoxes", ces religieux indiens qui cherchent à faire "revivre l'ancien" et qui préfèrent parler de «revivalisme», plutôt que de naissance comme le font les Britanniques.

Mais aucun des représentants de ces points de vue n'est capable, selon Roy, de faire prendre à l'Inde le chemin du progrès:

Neither the anxiety of the British Imperialists, nor the desire of the constitutional patriots, nor the fanaticism of the orthodox nationalists will be able to lead the rising Indian nation astray from the path marked out by those historical forces which determine human progress ${ }^{20}$.

Ce qu'il faut à présent, estime-t-il, c'est analyser le passé et le présent de l'Inde et voir son futur selon le point de vue du «matérialisme historique». Mais la tâche n'est pas aisée, juge-t-il, car l'historien est alors obligé de passer par le filtre de deux courants historiographiques fortement implantés dans les esprits de l'époque et liés aux perspectives

fondé sur le noble idéal d'assurer le bonheur et la prospérité pour la majorité du peuple» (M. N. Roy, «Bourgeois Nationalism»).

I8. Gandhi, par son attitude conciliante à l'égard du pouvoir britannique et son rejet de la rhétorique révolutionnaire, était en effet considéré par les marxistes comme un exemple typique de la "bourgeoisie indienne». Illustrant cette critique à l'aube de la Seconde Guerre mondiale, Léon Trotsky (1879-1940) a cette formule lapidaire à son égard: "The leader and prophet of this bourgeoisie is Gandhi. A fake leader and a false prophet!» (L. Trotsky, «An Open Letter to the Workers of India»).

19. Cf. M. N. Roy, India in Transition, p. 11: "The British ruling class is anxious to be the god-father of this child and has appointed the clever midwife of imperial Liberalism to help its birth.»

2o. Ibid., p. 12. 
vues ci-dessus, à savoir: 1) l'historiographie impérialiste qui s'intéresse plus à sa "mission civilisatrice» qu'aux conditions des peuples colonisés, et 2) l'historiographie indienne, qui "sacrifie les faits historiques sur l'autel du patriotisme ${ }^{21}$. Roy les estime, cela va sans dire, dépourvues d'objectivité ${ }^{22}$.

Comment alors se démarque-t-il de ces deux historiographies? Quel type de lecture propose-t-il à leur place pour expliquer la situation économique et matérielle de l'Inde? Dans le chapitre 6 de son essai, intitulé «Political Movement», l'auteur revient sur les événements sociaux et politiques qui ont précédé la période britannique. Il s'attelle tout d'abord à montrer qu'au "temps des invasions musulmanes" ${ }^{23}$, l'Inde était en partie sous régime féodal - parmi les Rajputs du Nord - et en partie, majoritairement d'ailleurs, divisée en royaumes théocratiques d'une part, patriarcaux d'autre part. Il écrit, par exemple:

Under such circumstances, national consciousness, embracing the entire population that inhabited the continent of India, was naturally an impossibility ${ }^{24}$.

Il poursuit sa critique de la conception nationaliste en insistant sur le fait que l'Inde n'est à ce stade qu'une "expression géographique», qu'elle n'a pu jusque-là contenir d'identité nationale. Plus précisément:

The extensive peninsula called India, is a mere geographical expression; it is very distinctly marked out from the mainland of Asia by physical barriers. But to hold that this geographical accident has been in itself sufficient to create a sense of national unity among the diverse communities inhabiting India, would be to misread the history of human evolution ${ }^{25}$.

Roy ne partage donc pas la conception des nationalistes hindous qui, en réaction aux «historiens impérialistes", attribuent à cette époque déjà l'existence d'une «nation hindoue» et évoquent le "passé glorieux» que

2I. Ibid., p. 13.

22. A ce stade (1922), la rhétorique asiatiste qui allait faire de l'Inde la porteuse de civilisation en Asie du Sud-Est n'a pas encore vu le jour. Elle apparaîtra quelques années plus tard avec la naissance de la Greater India Society (voir point 3 ci-après).

23. M. N. Roy, India in Transition, p. 148.

24. Ibid., p. 145.

25. Ibid., p. 147 sq. 
l'Inde aurait connu avant l'invasion des musulmans. Si Roy estime lui aussi que la population du sous-continent a pu effectivement souffrir de la présence de souverains étrangers, il ne lie pas pour autant cette situation à une incapacité inhérente qu'auraient eue les Indiens à forger une unité et une identité nationales, comme en concluent selon lui les historiens européens. Mais en même temps, il rejette tout aussi catégoriquement la position inverse des historiens nationalistes, qui prétendent que des liens culturels et religieux naturels soudaient les Indiens entre eux pour former justement "une unité nationale homogène» ${ }^{26}$. Aucune des deux explications communément défendues à son époque ne lui paraît correspondre aux véritables causes historiques ayant présidé à la prise de pouvoir par les Anglais sur le sous-continent.

Néanmoins, il n'échappe pas à une autre forme d'interprétation réactive vis-à-vis des historiens britanniques, en faisant des musulmans non pas des opposants religieux - la religion n'entre en rien dans son analyse historique - mais les précurseurs des capitalistes britanniques. C'est en raison des colonisateurs musulmans puis britanniques que le progrès des peuples indigènes fut freiné, dit-il. Il explique cela en ces termes:

The political state, imperial as well as provincial, was the apparatus of a dominant social class extraneous to the country. Its expression was mainly directed against the native feudal chiefs, an increase of whose power constituted a menace to the safety, - the very existence, of the Musalman authority. Thus the establishment of a more advanced form of political institution, instead of contributing towards, checked the social progress of the people ${ }^{27}$.

En bref, il interprète la conquête coloniale de l'Inde par les musulmans puis par les Britanniques comme le résultat d'une série de «causes et événements concomitants se développant de manière méthodique et répondant à des lois matérielles déterminées ${ }^{28}$. Ainsi, ce n'est ni la "perfidie» des colons britanniques, comme le maintiennent les nationalistes indiens, ni une «infériorité innée » des indigènes comme l'affirment les historiens britanniques, ni encore une "visée politique colonialiste», en ligne avec les discours orientalistes, qui doivent expliquer selon Roy

26. Ibid., p. 149.

27. Ibid., p. 147.

28. Ibid., p. 156. 
l'emprise britannique sur le sous-continent, mais une alliance de circonstances socio-économiques:

The English traders who came to the shores of India without any political pretensions, could eventually establish a great and mighty empire, because they happened to embody the social force which, in accordance with the imperious material laws determining all human progress, was next to assert itself over the political life of the country ${ }^{29}$.

Cet extrait permet de mettre en exergue deux aspects importants de la rhétorique de Roy sur l'histoire coloniale: premièrement, il rejette le point de vue selon lequel les Britanniques surent planifier le pouvoir économique et politique acquis au fil des siècles sur le sous-continent; deuxièmement, il insiste, à l'inverse, sur la prééminence du matérialisme historique pour expliquer les forces à l'œuvre dans cette partie du monde.

Roy admet toutefois que suite à cette première étape, en quelque sorte purement économique, les Britanniques ont eu l'intelligence de s'allier à la nouvelle classe commerçante indienne et à l'intelligentsia pour imposer leur emprise politique sur le peuple, et sur ces nouvelles élites par la même occasion ${ }^{30}$. Mais quoi qu'il en soit, pour lui, le jeu de forces qui parcourt l'Inde ressort toujours en dernière instance d'une série de facteurs socio-économiques; les questions religieuses n'entrent en aucune manière dans le schéma explicatif de Roy ${ }^{31}$. Cette position va clairement à l'encontre des conceptions dominantes de l'époque qui voulaient voir dans l'Asie en général et l'Inde en particulier le lieu d'un modèle d'expression avant tout spirituelle.

29. Ibid., p. 156 sq.

30. Ibid., p. 157: "The British East India Company succeeded in establishing its political domination over India with the help of, and subsequently at the cost of, the native trading class which, together with the intelligencia, constituted the progressive and objectively most revolutionary factor of Indian society in the middle and latter parts of the eighteenth century."

3I. Dans le «Programme for the Indian National Congress» qu'il rédige et envoie à diverses personnes et organisations en Inde en décembre 1922, Roy établit un programme de «libération nationale» en vingt points. Alors que le premier objectif listé est l'indépendance complète du pays, suivi par une série d'objectifs socio-économiques, le premier point mentionnant expressément la question religieuse n'intervient qu'en antépénultième position et dit: "The State will be separated from all religious creeds, and the freedom of belief and worship will be guaranteed.» (M. N. Roy, "A Programme for the Indian National Congress »). 


\section{Roy et les discours asiatistes}

Quelles étaient ces conceptions et comment peut-on situer la position de Roy parmi les différentes réponses indiennes au regard colonial et orientaliste? L'article "Imagining Asia in India: Nationalism and Internationalism (ca 1905-1940)" de Carolien Stolte et Harald FischerTiné sera utile ici à notre propos. Dans cet article, les auteurs délimitent et développent trois types de rhétoriques asiatistes présents chez les auteurs indiens de la première moitié du $\mathrm{XX}^{\mathrm{e}}$ siècle ${ }^{32}$ :

a) l'Asie conçue comme antithèse spirituelle à l'Europe - les auteurs présentent comme figure de proue de cette conception Rabindranath Tagore;

b) l'Asie vue comme "India Magna", en d'autres termes l'idée que la "Grande Inde» a fortement influencé l'Asie - idée essentiellement soutenue par Kalidas Nag, l'auteur de Greater India (1926);

c) et la "Jeune Asie», présentant le continent comme le lieu d'une modernité supérieure - et dont les principaux "prophètes» en Inde (selon le mot des auteurs) furent Benoy Kumar Sarkar et Taraknath Das.

Le premier type de rhétorique est probablement le plus connu en dehors de l'Asie et celui qui vient en premier à l'esprit lorsqu'on pense aux réactions indiennes, ou asiatiques, à la présence coloniale. Pour l'Inde, le discours de Vivekānanda (1863-1902), mais aussi celui de la Société Théosophique, est certainement le plus représentatif de cette emphase sur "l'Inde spirituelle». Toutefois, certains penseurs indiens ont élargi cette idée au continent dans son entier. C'est notamment le cas de Rabindranath Tagore (1861-1941) qui, dans un message délivré durant sa première visite au Japon en 1916, "était d'avis que l'Occident sécularisé ne pouvait se réformer de l'intérieur. Ce rôle historique et mondial devait être réservé à l'Asie, notamment à sa principale puissance émergente, le Japon " ${ }^{33}$. Stolte et Fischer-Tiné soulignent toutefois que

32. C. Stolte, H. Fischer-Tiné, «Imagining Asia in India», p. 75 sq. Ces auteurs définissent globalement les discours "asiatistes» comme "discourses and ideologies claiming that Asia can be defined and understood as a homogenous space with shared and clearly defined characteristics» (ibid., p. 65).

33. Ibid., p. 77. 
Tagore ne manquera pas de condamner très fermement l'impérialisme et le nationalisme japonais ${ }^{34}$. Par la suite, c'est Gandhi, cible privilégiée de Roy comme on l'a déjà dit, qui reprendra le flambeau de "l'unité spirituelle» de l'Asie, à la différence près toutefois qu'il percevait pour sa part "toute implication avec l'Asie comme une nécessité regrettable» ${ }^{35}$.

A la lecture des textes de Roy, il paraît évident que celui-ci éprouvait un dédain radical pour toute politique nationaliste, de quelque origine qu'elle fut; sur ce point il peut donc être comparé à Tagore ${ }^{36}$. Il est par contre exclu de l'assimiler plus largement à la rhétorique de Tagore, tant la conception spiritualiste de celui-ci lui paraissait irrecevable comme réponse à la question coloniale. Une critique sans ambages de cette attitude qu'il qualifiait "d'impérialisme spirituel» fait justement partie de ses arguments contre le discours de Vivekānanda dans India in Transition:

He preached that Hinduism, not Indian nationalism, should be aggressive. His nationalism was a spiritual imperialism. [...] This romantic vision of conquering the world by spiritual superiority electrified the young intellectuals, whose desperate economic position made them restive ${ }^{37}$.

Roy rejeta en bloc le discours orientaliste de l'entre-deux-guerres qui voulait voir dans le «spiritualisme indien» le modèle salvateur du monde ${ }^{38}$.

34. Sur Tagore et le rôle de Calcutta dans le discours panasiatiste, voir M. R. Frost, " "That Great Ocean of Idealism" "; sur Tagore et sa réception en Asie, voir S. N. Hay, Asian Ideas of East and West.

35. C. Stolte, H. Fischer-Tiné, "Imagining Asia in India», p. 80.

36. Cf. par exemple M. N. Roy, Selected Works of M. N. Roy, vol. 2, p. 225 ( On Patriotism ", lettre datée du 12 juin 1923): «A movement which is based only on patriotism cannot go very far in these days. Pure Indian patriotism smacks of reaction, and produces Gandhis and Arabindas [i.e. Aurobindo], about whom you have no more illusions"; ou ibid., p. 237 ("On the Duty of the Revolutionary Intellectuals», lettre datée du 15 août 1923): «It is stupidity or sheer hypocrisy to say that our nationalism will be different from European nationalism. Nationalism is always aggressive, directly or indirectly."

37. M. N. Roy, India in Transition, p. 192 sq. En ce sens, et même si le regard de Roy sur la question spirituelle changera quelque peu par la suite, il paraît erroné de faire un «rapprochement étroit» avec Vivekānanda comme le fait D. G. Dalton, «M. N. Roy and Radical Humanism ", p. 158.

38. Quelques années plus tard, après sa sortie de prison suite à son retour en Inde, il utilisera un langage encore plus fleuri pour dénoncer cette conception de l'Inde 
Partageant son point de vue, Evelyn Trent, sa première femme, offre un exemple parlant de ce refus dans l'extrait suivant, dirigé contre la perception idyllique de Gandhi par Roman Rolland (1866-1944) :

India's triumph will be a world triumph of the forces of light over darkness, of spirit over matter, of God over Satan. With such a conception of the Indian struggle for freedom we have nothing to do; it embodies the exaggerated subjectivism of the disillusioned post-war intellectual, flying to the realm of metaphysics to escape from the cruel logic of facts and realities ${ }^{39}$.

Deux ans plus tard, Roy dénoncera de manière encore plus virulente la vision dichotomique de Rolland opposant l'Asie à l'Europe, attribuant à ce dernier la crainte d'une insurrection asiatique et africaine et d'un "péril» potentiel pour l'Europe. A cette division géographique et politique, Roy opposera une lecture du monde basée sur la dichotomie marxiste divisant la société en classes prolétaires et bourgeoises:

We suggest to M. Rolland to try to change his categories of thought. Instead of thinking so exclusively in the terms of Asia and Europe, he might try to think in the terms of wage-slaves and capital, of the proletariat and the bourgeoisie. He will then see that his imagined Asiatic Peril is neither distinctly Asiatic nor is it a peril except to capitalists (in both Europe and Asia) ${ }^{40}$.

Le deuxième type d'asiatisme souligné par Stolte et Fischer-Tiné est cette fois lié à cette forme de nationalisme qui fit de l'Inde le "porteur de la civilisation" en Asie du Sud-Est et qui se fit connaître en tant qu'organisation sous le nom de Greater India Society ${ }^{41}$. Parmi ses membres les plus influents, Kalidas Nag (1891-1966) et P. C. Bagchi

(M. N. Roy, Fragments of a Prisoner's Diary, vol. 2, p. VII) : "The belief in India's spiritual message to the materialist West is a heady wine. It is time to realise that the pleasant inebriation offered a solace to proud intellectuals with inferiority complex.»

39. E. Roy, «Mahatma Gandhi».

40. M. N. Roy, Selected Works of M. N. Roy, vol. 2, p. 362 ("Mr Rolland and the "Asiatic Peril" ", publié dans The Masses of India, I/7, juillet 1925).

4I. Sur la Greater India Society et sur la relation de ses membres avec l'indianisme français, voir de S. Bayly, "Imagining "Greater India”" et "India’s "Empire of Culture"». 
(1898-1956) ${ }^{42}$ furent des orientalistes formés à Paris par Sylvain Lévi (1863-1935) et Jean Przyluski (1885-1944). C'est auprès de ceux-ci qu'ils assimilèrent l'idée que l'Inde hindoue et bouddhique avait autrefois connu son heure de gloire et avait su "propager ses savoirs culturels, spirituels et matériels» à travers toute l'Asie ${ }^{43}$. Cette idée d'une «Grande Inde» essaima dans toutes les classes éduquées de l'Inde et se popularisa particulièrement parmi les organisations hindoues comme la Hindu Mahasabha ${ }^{44}$ : «Le point central de l'asiatisme de la Hindu Mahasabha consistait à faire de l'Asie un continent hindou et bouddhiste" ${ }^{45}$.

S'il y a bien un discours auquel Roy ne peut être associé, c'est celui-ci. Roy s'est toujours clairement distancié de toute forme de nationalisme indien, encore plus hindou. Un aspect, néanmoins, nous permet de ne pas complètement dissocier Roy des nationalistes, malgré ce qui vient d'être dit. C'est le fait qu'il a insisté, tout comme les nationalistes, sur le rôle primordial que pouvait et devait jouer l'Inde dans la lutte des pays colonisés contre les puissances coloniales. Mais, une fois encore, les réponses apportées furent nettement distinctes dans un discours et dans l'autre. Si Roy accordait à «l'Orient» en général et à l'Inde en particulier un rôle majeur dans l'histoire mondiale de ce début de XXe siècle, c'était en raison de ce qu'il estimait être la force exemplaire de son mouvement révolutionnaire et non pour un quelconque motif religieux ou culturel. Comme nous l'avons vu ci-dessus, par rapport au premier type de rhétorique, Roy ne s'est pas inscrit en faux contre toute forme d'universalisme. Ce qui distingue son discours des autres, et notamment de celui de la Greater India Society, est la primauté accordée aux critères socioéconomiques, au détriment des arguments nationalistes, religieux et culturels. A ce titre, sa rhétorique se démarque complètement des divers discours orientalistes et non seulement de l'orientalisme britannique, comme le firent les partisans de la «Grande Inde» ${ }^{46}$.

42. Deux des membres fondateurs de la Greater India Society, fondée à Kolkata en 1926.

43. C. Stolte, H. Fischer-Tiné, "Imagining Asia in India», p. 83.

44. "La grande assemblée des hindous», parti nationaliste hindou fondé en 1915 pour défendre les intérêts des hindous au sein du Congress, qui fit place plus tard au Bharatiya Jana Sangh, puis à l'actuel Bharatiya Janata Party (BJP), le «Parti du peuple indien".

45. C. Stolte, H. Fischer-Tiné, "Imagining Asia in India», p. 85.

46. S. Bayly, "Imagining "Greater India”", p. 710 sq. 
Reste le troisième type de rhétorique présentée dans l'article de Stolte et Fischer-Tiné. Selon les auteurs, cette rhétorique se distingue des deux précédentes en ce sens qu'elle "résista fondamentalement à l'axiome de la spiritualité “inhérente" à l'Asie" ${ }^{47}$. Pour son représentant le plus célèbre, le Bengali Benoy Kumar Sarkar (1887-1949), la soi-disant supériorité de l'Occident sur l'Asie n'était qu'une pure construction historique, tout comme le mythe d'une différence de civilisation, purement issu selon lui d'une lecture orientaliste des anciens textes de l'Asie, déconnectée de leur contexte de production ${ }^{48}$. Pour Sarkar, anti-impérialiste radical, et ses compagnons de "Young Asia», la réponse à l'Occident ne devait pas passer par la voie de la spiritualité, mais reconnaître et utiliser au contraire toutes les sciences et techniques de l'Europe et de l'Amérique, comme avait d'ailleurs commencé à le faire le Japon. C'est ce qu'il explique dans "The Futurism of Young Asia", un article publié une première fois en 1918 dans la revue International Journal of Ethics et issu d'une conférence donnée à la Clark University de Worcester une année plus tôt ${ }^{49}$. Voici, finalement, une perspective qui s'approche de celle de Roy, en tout cas par leur refus commun de voir en l'Inde, et l'Asie, une simple réponse spirituelle à l'Occident. Un rapprochement entre les deux auteurs d'autant plus vraisemblable que Sarkar fut le maître de Roy au Bengal Technical Institute en $1907^{50}$. Une étude comparée et détaillée des points de convergence et de divergence entre les deux Bengalis apporterait certainement une lumière supplémentaire à l'histoire des relations politiques, économiques et intellectuelles entre ces deux parties du monde; cela déborderait toutefois le cadre de cette contribution et nécessiterait une autre recherche.

\section{Conclusion}

Avant de conclure, un aspect essentiel de la situation géographique et intellectuelle de Roy nécessite d'être mentionné en relation avec le thème

47. C. Stolte, H. Fischer-Tiné, «Imagining Asia in India», p. 88.

48. Ibid., p. 89.

49. B. K. Sarkar, "The Futurism of Young Asia». L'article est ensuite publié avec d'autres essais "on the relations between the East and the West" sous forme de livre en 1922 à Berlin, la même année et au même endroit donc que India in Transition de Roy. 5o. K. Manjapra, M. N. Roy, p. 20. 
de cette publication, afin de pouvoir ajouter un éclairage supplémentaire à son parcours atypique. Il s'agit du fort lien existant entre l'appartenance d'un individu au "centre" - et le fait d'avoir ainsi un accès privilégié à l'information - et la possibilité de détenir par ce biais-là une certaine forme de pouvoir. En ce sens, Roy doit clairement être associé à une situation de pouvoir, puisqu'il se trouvait à double titre au cœur même des centres décisionnels et idéologiques de son époque, à savoir: l'Europe, et plus particulièrement dans son cas Berlin, foyer d'innombrables mouvements intellectuels, politiques et culturels du début du $\mathrm{XX}^{\mathrm{e}}$ siècle, et l'Internationale communiste, foyer de l'idéologie marxiste, dont il occupa pour un temps tous les postes clés ${ }^{51}$. Il avait ainsi non seulement accès à toutes les informations capitales de son temps, qu'elles proviennent d'Europe ou d'Inde - de nombreux informateurs ou émigrés indiens le tenaient au courant des événements du sous-continent mais il était, qui plus est, lui-même en mesure de créer et de diffuser ce savoir grâce à ses fonctions politiques et à ses constantes publications en de nombreuses langues.

Cette dernière réflexion en tête, reprenons les différents éléments importants de l'article pour interroger les notions de «centre» et de «marges» dans un cas de figure comme celui de Roy. Nous avons vu, pour commencer, que Roy, au même titre que les nationalistes indiens, a offert une réponse personnelle au regard porté par les historiens britanniques («orientalistes» ou «impérialistes») sur l'Inde. Il a ainsi contribué à la vaste réponse des marges au discours du centre. Mais, contrairement à eux, il n’a pas "inventé» un passé indien glorieux, unifié, basé sur de grandes valeurs spirituelles et atemporelles, en opposition au supposé matérialisme occidental. Au contraire, il a repris le leitmotiv du progrès social et économique des grands discours occidentaux, que ceux-ci fussent de nature évolutionniste, impérialiste, orientaliste ou marxiste, pour l'appliquer à la situation indienne en particulier et à celle de tous les pays et peuples colonisés en général, sans pour autant privilégier l'idée d'une unité, d'une essence asiatique. De ce point de vue là, il s'est réapproprié le centre pour expliquer les marges. Par ailleurs, le constat s'impose qu'il a adopté, lui aussi, une perspective et un discours à visée

5I. Il faudrait ajouter à cela le fait qu'il était originaire du Bengale et qu'il ait grandi à proximité de Kolkata puis dans la ville même, alors capitale de l'Empire britannique des Indes et un des centres les plus cosmopolites de l'océan indien. 
universaliste - qui plus est lorsqu'il s'est tourné, de retour en Inde, vers la philosophie humaniste et rationaliste du Radical Humanism. Il s'est ainsi approprié le langage du centre, du pouvoir, mais en privilégiant une région (l'Inde et plus précisément Dehra Dun, au pied de l'Himalaya) perçue comme appartenant aux "marges» dans le contexte colonial, mais aussi dans les années suivant l'Indépendance.

La question qui demeure est la suivante: peut-on échapper, même avec un parcours aussi atypique et désorientant que celui de Roy, à la tension bipolaire du centre et de la périphérie (ou des marges) ? Peut-on l'assouplir, l'étendre pour y inclure un troisième terme? Un exemple possible de "décentrement" hors de l'opposition «centre-périphérie» pourrait être fourni par la situation de Tachkent: comme nous l'avons vu ci-dessus, cette ville a pu fonctionner comme un réel pôle de convergence et de rencontres vers la fin du XIX et le début du XX $\mathrm{XX}^{\mathrm{e}}$ siècle, alors que l'on retient d'elle l'image d'une ville et d'une région fort éloignées des capitales et centres de pouvoir de l'époque, représentés par des villes comme Londres, Moscou ou Kolkata.

S’il paraît difficile d'éviter le schéma "marges-centre» qui semble s'imposer de lui-même, même dans ce cas de figure, tout au moins l'étude d'un auteur comme M. N. Roy présente le double avantage de mettre en lumière la nature dynamique et mouvante de cette relation et de devoir repenser les différentes postures idéologiques qui avaient cours en Inde durant la première moitié $\mathrm{du} \mathrm{XX}^{\mathrm{e}}$ siècle, rappelant ainsi que tous les Indiens ne partageaient pas ce topos orientaliste de l'Inde spirituelle s'opposant au monde occidental scientifique et matérialiste.

Nicola Pozza

Université de Lausanne 


\section{BIBLIOGRAPHIE}

BAYLY, Susan, «Imagining "Greater India”: French and Indian Visions of Colonialism in the Indic Mode", Modern Asian Studies, 38/3 (2004), p. 703-744.

—, «India's "Empire of Culture": Sylvain Lévi and the Greater India Society», in Sylvain Lévi (1863-1935): Etudes indiennes, histoire sociale, éd. par Lyne Bansat-Boudon, Roland Lardinois, Turnhout, Brepols, 2007, p. 193-212.

Bhattacharya, Swapna, "Bengal and Germany: Some Aspects of Political, Economic and Intellectual Encounter", en ligne: <http://www.india.diplo.de/contentblob/3184072/ Daten/2137509/download_datei_bengal_germany.pdf> (consulté le 21.09.2013).

Boquerat, Gilles, «Du bond en avant au retour en arrière, évolution de la perception indienne de l'Asie centrale au cours du XX $\mathrm{XX}^{\mathrm{e}}$ siècle", Cahiers d'Asie centrale, 1/2 (1996), p. 283-296.

CARR, Barry, "Marxism and Anarchism in the Formation of the Mexican Communist Party, 1910-19", The Hispanic American Historical Review, 63/2 (1983), p. 277-305.

Chouman, A. P. S., Singh, Dinesh Kumar, "M. N. Roy and Marxism», The Indian Journal of Political Science, 66/3 (2005), p. 633-648.

Dalton, D. G., "M. N. Roy and Radical Humanism: The Ideology of an Indian Intellectual Elite", in Elites in South Asia, ed. by Edmund Leach, S. N. Mukherjee, Cambridge, Cambridge University Press, 1970, p. 152-171.

Frost, Mark Ravinder, "That Great Ocean of Idealism”: The Tagore Circle and the Idea of Asia, 1900-1920", in Indian Ocean Studies: Cultural, Social and Political Perspectives, ed. by Shanty Moorthy, Ashraf Jamal, New York/London, Routledge, 2010, p. 251-279.

Goebel, Michael, «Una biografía entre espacios: M. N. Roy, del nacionalismo indio al comunismo mexicano", Historia Mexicana, 62/4 (2013), p. 1457-1493.

Haithcox, John Patrick, Communism and Nationalism in India: M. N. Roy and Comintern Policy, 1920-1939, Princeton, Princeton University Press, 1971. 
Hay, Stephen N., Asian Ideas of East and West: Tagore and his Critics in Japan, China, and India, Cambridge (Mas.), Harvard University Press, 1970.

Karnik, V. B., M. N. Roy: Political Biography, Bombay, Nav Jagriti Samaj, 1978.

Kaviraj, Sudipta, "The Heteronomous Radicalism of M. N. Roy", in Political Thought in Modern India, ed. by Thomas Pantham, Kenneth L. Deutsch, New Delhi/Beverly Hills/London, Sage, 1986, p. 209-235.

Manjapra, Kris, M. N. Roy: Marxism and Colonial Cosmopolitanism, London/New York/New Delhi, Routledge, 2010.

Minault, Gail, The Khilafat Movement: Religious Symbolism and Political Mobilization in India, New York/Guildford, Columbia University Press, 1982.

Nath, Ramendra, "Manbendra Nath Roy (1887-1954)», Internet Encycopedia of Philosophy, en ligne: <http://www.iep.utm.edu/ roy_mn/> (consulté le 21.09.2013).

Qureshi, M. Naeem, Pan-Islam in British Indian Politics: A Study of the Khilafat Movement, 1918-1924, Leiden/Boston/Köln, Brill, 1999.

Roy, Evelyn, "Mahatma Gandhi: Revolutionary or CounterRevolutionary? A Reply to Romain Rolland and Henri Barbusse», Labour Monthly, 5/3 (1923), en ligne: <http://www.marxists. org/archive/roy-evelyn/articles/1923/gandhi_rev_counter.htm> (consulté le 18.02.2014).

Roy, M. N., India in Transition, with collaboration of Abani Mukherji, Genève, Edition de la Librairie J. B. Target, 1922.

-, "A Programme for the Indian National Congress", The AdvancedGuard, (1 décembre 1922), en ligne: <http://www.marxists.org/ archive/roy/1922/12/national-congress-program.htm> (consulté le 18.02.2014).

—, "Bourgeois Nationalism», Vanguard, 3/1 (1923), en ligne: <http:// www.marxists.org/archive/roy/1923/08/15a.htm> (consulté le 18.02.2014).

—, "On the Duty of Revolutionary Intellectuals", Political Letters, Zurich, The Vanguard Bookshop (1924), en ligne: <http:// www.marxists.org/archive/roy/1923/08/15.htm> (consulté le 18.02.2014). 
—, Fragments of a Prisoner's Diary, vol. 2, India's Message, Calcutta, Renaissance Publishers, 1950.

—, Selected Works of M. N. Roy, vol. 1, 1917-1922, ed. by Sibnarayan Ray, New Delhi [etc.], Oxford University Press, 1987.

—, Selected Works of M. N. Roy, vol. 2, 1923-1927, ed. by Sibnarayan Ray, New Delhi [etc.], Oxford University Press, 2000.

SARKar, Benoy Kumar, "The Futurism of Young Asia", International Journal of Ethics, 28/4 (1918), p. 521-541.

Stolte, Carolien, Fischer-Tiné, Harald, "Imagining Asia in India: Nationalism and Internationalism (ca. 1905-1940)», Comparative Studies in Society and History, 54/1 (2012), p. 65-92.

Trotsky, Leon, "An Open Letter to the Workers of India", New International, 5/9 (1939), p. 263-266. En ligne: <http://www. marxists.org/archive/trotsky/1939/07/india.htm> (consulté le 21.09.2013).

Vigreux, Jean, «Manabendra Nath Roy (1887-1954), “représentant des Indes britanniques" au Komintern ou la critique de l'impérialisme britannique», Cahiers d'histoire. Revue d'histoire critique, 111 (2010), p. 81-95. En ligne: <http://chrhc.revues.org/2075> (consulté le 21.09.2013). 
